\title{
t-SNARE Transmembrane Domain Clustering Modulates Lipid Organization and Membrane Curvature
}

\author{
Satyan Sharma*, ${ }^{*}$ and Manfred Lindau ${ }^{\dagger, \S}$ \\ ${ }^{\dagger}$ Laboratory of Nanoscale Cell Biology, Max-Planck-Institut für Biophysikalische Chemie, Göttingen 37077 Germany \\ ${ }^{\S}$ School of Applied and Engineering Physics, Cornell University, Ithaca, New York 14850, United States
}

Supporting Information

ABSTRACT: The t-SNARE complex plays a central role in neuronal fusion. Its components, syntaxin-1 and SNAP25, are largely present in individual clusters and partially colocalize at the presumptive fusion site. How these protein clusters modify local lipid composition and membrane morphology is largely unknown. In this work, using coarse-grained molecular dynamics, the transmembrane domains (TMDs) of t-SNARE complexes are shown to form aggregates leading to formation of lipid nanodomains, which are enriched in cholesterol, phosphatidylinositol 4,5-bisphosphate, and gangliosidic lipids. These nano-domains induce membrane curvature that would promote a closer contact between vesicle and plasma membrane.

$\mathrm{I}_{\mathrm{a}}^{\mathrm{n}}$ neuronal cells, the soluble $N$-ethylmaleimide-sensitive factor attachment protein receptor (SNARE) complex, consisting of the vesicle associated synaptobrevin 2 (syb2), also known as VAMP2, and the plasma membrane t-SNARE complex of syntaxin 1 (stxl) and synaptosome associated protein 25 (SNAP25) constitute the minimal fusion machinery. ${ }^{1}$ Syb2 and stxl are anchored to the respective membranes via their transmembrane domains (TMDs). SNAP25 is anchored via a palmitoylated cys-rich domain. The cytoplasmic SNARE domains of syb2 and the t-SNAREs zipper-up to form a tight complex bridging the synaptic vesicle and plasma membranes and contribute energy necessary for fusion. ${ }^{1,2}$

Fusion involves a close interplay between the membrane lipids and fusion proteins. ${ }^{3-5}$ Presumably, one important role of the lipids is in formation of functional domains that sequester fusion proteins. The t-SNAREs stx1 and SNAP25 are organized in clusters, which partially overlap, and are sites of vesicle docking and fusion. ${ }^{6-12}$ In PC12 cells, t-SNARE clusters contain similar copy numbers $(\sim 50-70)$ stx1 and SNAP25 molecules that presumably exist as t-SNARE binary complexes at sites of docked vesicles. ${ }^{7}$ Phosphoatidylinositol 4,5-bisphosphate (PIP2) and cholesterol have been shown to be important for clustering of $t$ SNAREs. ${ }^{6,13-15}$

To determine how clustering of t-SNAREs controls lipid organization of the plasma membrane, we performed coarsegrained molecular dynamics (CGMD) simulations using the MARTINI force field. ${ }^{16}$ The structural organization of the SNARE domains in a prefusion t-SNARE complex is not known. The C-terminal regions of the t-SNAREs interact most intimately with the membrane lipids; thus, only the stx1 TMD and the adjacent juxtamembrane domain in a complex with the interacting regions of SNAP25 were chosen.

Since the molecular structure of t-SNARE clusters is unknown, we used self-assembly simulations to study spontaneous clustering of 12 copies of t-SNARE C-terminal fragments (stx1 A247-I288, SNAP25 A74-S98, and A195-G206 with the side chains of $\mathrm{C} 85, \mathrm{C} 88, \mathrm{C} 90$, and $\mathrm{C} 92$ replaced by palmitoyl chains). The initial structure of a monomeric t-SNARE complex was taken from a crystal structure of the SNARE complex, 3HD7 (Figure 1a). ${ }^{17}$ The TMDs were initially placed on a regular grid such that the minimum distance between any two atoms of the two neighboring t-SNAREs was at least $2.0 \mathrm{~nm}$. This arrangement of proteins was then inserted into a box containing randomly placed lipids with a composition based on that of purified synaptic plasma membranes of adult rat brain (Figure 1b, Table S1). ${ }^{18}$ Briefly, the system consisted of $12 \mathrm{t}$-SNARE fragments, 1455 lipids, 32734 waters, and $558 \mathrm{Na}^{+}$and $355 \mathrm{Cl}^{-}$ ion beads. The system was subjected to a $200 \mathrm{~ns}$ long selfassembly simulation, allowing a membrane with physiological asymmetric leaflet composition to self-assemble, ${ }^{19}$ with proteins restrained along the $x$ - and $y$-directions. After membrane selfassembly (Figure 1c), three independent $5 \mu$ s long production runs were started without any restraints ( $\operatorname{Sim} 1$ to 3 ). Additional details of the simulation methodology are given in the Supporting Information (SI).

During the course of individual simulations, the t-SNARE fragments diffused in the membrane and quickly associated to form a single large cluster (Figures $1 \mathrm{~d}, 2 \mathrm{a}$, and $\mathrm{S} 1$ ). The analysis of inter-t-SNARE protein contacts showed that the most prominent contact face is formed between two C-terminal halves of the stxl TMDs from adjacent t-SNAREs. Additional interactions were found between cys-rich linker domains of SNAP25-SN1. While no major inter t-SNARE interactions were observed between SNAP25-SN1 and stx1, SNAP25-SN2 showed many contacts with SNAP25-SN1 as well as stx1 (Figure S2).

Analysis of protein-lipid interactions revealed that, already during the self-assembly, PIP2 and gangliosides (GM; monosialotetrahexosylganglioside (GM1) and monosialodihexosylganglioside (GM3)) begin to associate with t-SNAREs. As the t-SNAREs cluster, PIP2 and GM cocluster with them (Figures 2a, S1, and S4). Figure $2 \mathrm{~b}$ shows a quantitative analysis of the local enrichment of various lipid species in the vicinity of the protein. For this, the lipid composition within $0.8 \mathrm{~nm}$ of the protein was compared to the bulk composition of the

Received: October 6, 2017

Published: December 12, 2017 


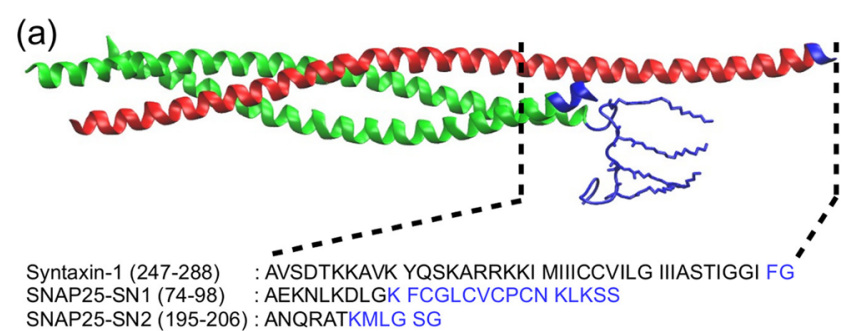

(b)
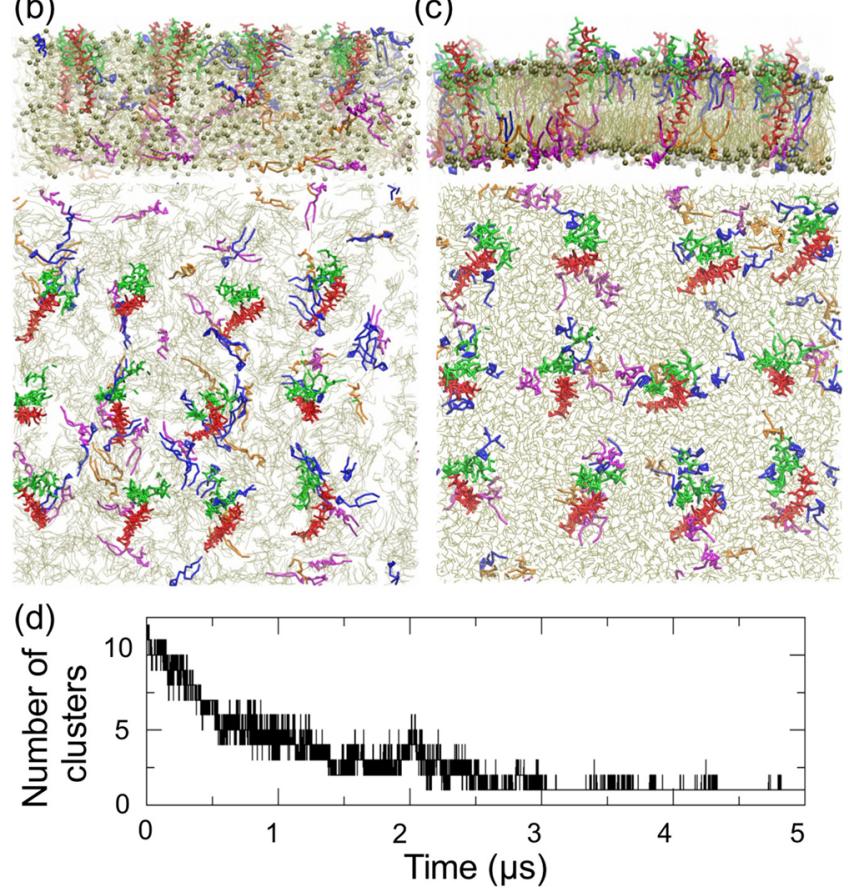

Figure 1. (a) Representation of t-SNARE complex from the crystal structure (3HD7). Missing residues were modeled (shown in blue) along with the palmitoyl chains (in sticks). The sequence of the region of $\mathrm{t}$-SNARE used in this study is indicated by dashed lines. $(\mathrm{b}, \mathrm{c})$ Twelve $\mathrm{t}-$ SNARE copies placed in random lipids (PIP2, blue; GM1, magenta; GM3, orange; rest lipids, tan) (b) before and (c) after a 200 ns selfassembly simulation (side-view, top panels; top-view, bottom panels). tSNAREs are sown in stick representation (stx1, red; SNAP25, green). (d) Evolution of the number of t-SNARE clusters from one of the simulations.

corresponding leaflet. The analysis clearly reveals local enrichment of PIP2 and GM lipids in the vicinity of the protein. Note that the membrane was formed by self-assembly from asymmetrically mixed lipids to obtain an asymmetric distribution of lipids in the two membrane leaflets. The resulting asymmetry was $\sim 70-90 \%$, depending on the specific lipid, independent of the presence of the t-SNAREs (Tables S1, Figure S5). An imperfect leaflet asymmetry resembles the actual situation in plasma membranes. $^{20}$

To identify the PIP2 interacting sites of the proteins, the average number of contacts of specific protein residues with headgroup phosphates of PIP2 was determined (Figure 3a). PIP2 formed favorable interactions with the basic amino acids 256 and $260 \mathrm{~K}$ of stx1, as previously reported. Unexpectedly, we also found PIP2 interactions with basic residues of SNAP25, namely, 79K, $83 \mathrm{~K}, 198 \mathrm{R}$, and $201 \mathrm{~K}$. A recent study indicates that this PIP2 interacting domain of SNAP25 may be important for its interaction with the membrane prior to palmitoyation. ${ }^{21}$ It is also possible that PIP2-SNAP25 interactions could facilitate the formation of binary stx1-SNAP25 t-SNARE complexes.

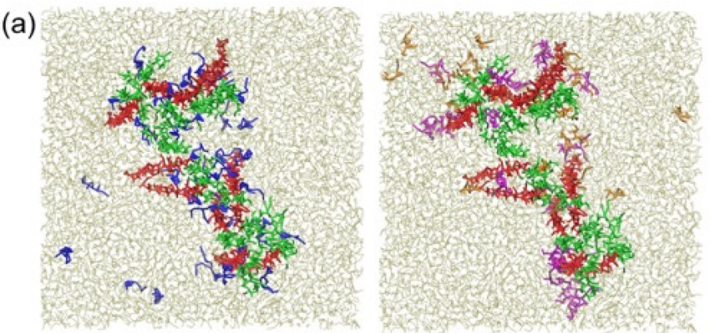

(b)

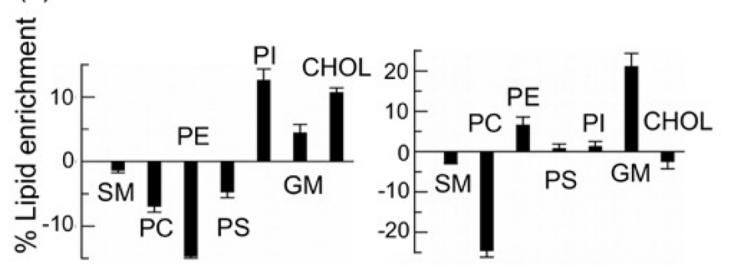

(c)

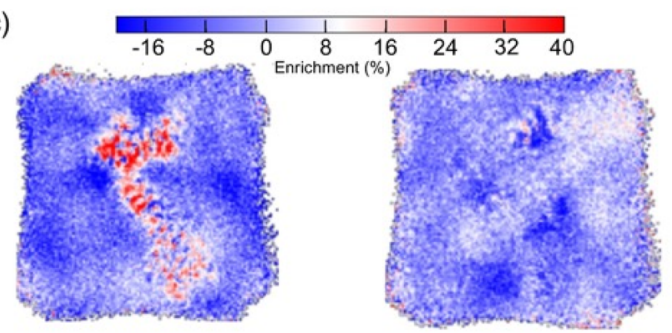

Figure 2. (a) Snapshot at $5 \mu$ s showing PIP2 clusters at the IC leaflet (left) and GM lipids at the EC leaflet (right) (PIP2, blue; GM1, magenta; GM3, orange) with the t-SNARE cluster (stx1, red; SNAP25, green). (b) Local lipid enrichment (mean $\pm \mathrm{SD}, n=3$ simulations) near t-SNARE complexes in the IC (left) and EC (right) leaflets. (c) Cholesterol enrichment averaged over the last $200 \mathrm{~ns}$ in the IC (left) and EC (right) leaflets.
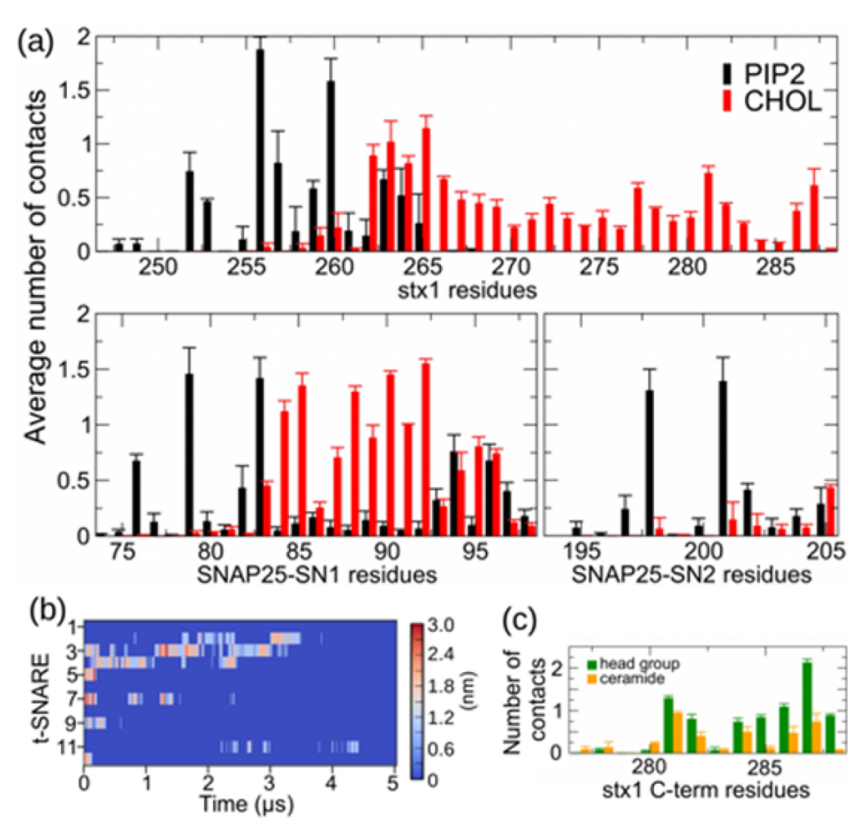

(c)

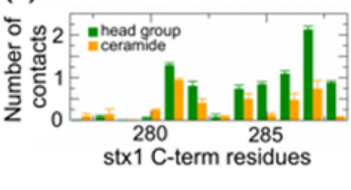

Figure 3. (a) Average number of contacts of specific stxl (upper panel) and SNAP25-SN1 and SNAP25-SN2 residues (lower panel) with PIP2 (black) and cholesterol (red). (b) Minimum distance between stx1 TMD and GM during the course of simulation. (c) Average number of contacts of stx1 TMD with GM. (a,c) Mean \pm SD $(n=3)$.

At the EC leaflet, GM was found to colocalize with the protein cluster (Figures 2a,b). The time evolution of minimum distance 
between the individual t-SNARE complexes and GM lipids shows that all complexes interact tightly with GM (Figure $3 b$ ). The GM headgroup interacts primarily with the charged Cterminus and polar residues of the C-terminal half of the stx 1 TMD.

The average cholesterol distributions in the IC and EC leaflets were $\sim 38 \%$ and $45 \%$, respectively, with the remaining cholesterol located in the hydrophobic core (Table S1). Cholesterol molecules showed a flip-flop rate constant of $8.38 \pm 0.01 \times$ $10^{6} \mathrm{~s}^{-1}$, close to the value observed in a previous simulation study on an asymmetric plasma membrane $\left(6.53 \pm 0.01 \times 10^{6} \mathrm{~s}^{-1}\right){ }^{22}$

Interestingly, all three independent CG-MD simulations showed in the IC leaflet a high local enrichment of cholesterol in the t-SNARE cluster (Figures $2 \mathrm{c}$ left, S6 top panels). SNAP25 interacts with cholesterol specifically in its palmitoylated region (residues 85-92) (Figure 3a). The EC leaflet of the membrane shows weakly enriched cholesterol domains (Figure 2c, right). Cholesterol interactions were also observed with stxl residues in region 261A-265 K, which are located near the surface of the IC leaflet. Other stx1 residues along the TMD showed interactions with cholesterol present in the inner hydrophobic core of the membrane.

It is well-known that the local enrichment of cholesterol in one leaflet with GM in the opposite leaflet induces membrane curvature due to the inherent properties of these lipids. ${ }^{23}$ To assess the changes in membrane morphology due to the $\mathrm{t}$ SNARE mediated lipid clustering, we carried out a $10 \mu$ s long simulation of a system with the number of lipids and C-terminal t-SNARE fragments quadrupled (SimL1). In the larger system, clustering of $t$-SNAREs, local cholesterol enrichment, and interactions of PIP2 and GM lipids with the t-SNAREs (Figures S7-S9) were all consistent with the simulations for the smaller membrane patch described above.

Visual inspection of snapshots showed that membrane undulation appeared within $250 \mathrm{~ns}$ of the simulation. As the simulation progressed and proteins aggregated to form clusters, one prominent region of the curvature appeared stably associated with the largest t-SNARE cluster. The GM lipid clusters are located in this region, associated with the C-terminus of stxl (Figure 4a). The membrane is bulged outward in the cluster

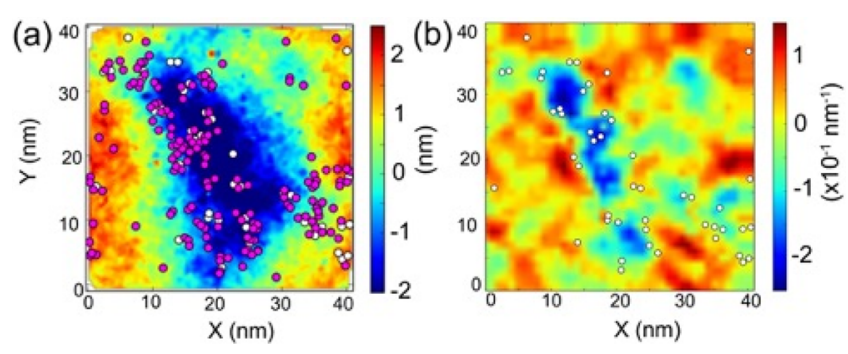

Figure 4. Membrane morphology during the final $20 \mathrm{~ns}$ of simulation SimL1. (a) Top view of the EC leaflet colored according to the deviation of the EC lipid head groups from their mean $z$-position. Also shown are the stx1 C-termini (white) and centers of mass of GM lipids (magenta). (b) Total curvature of the membrane.

center creating negative curvature and inward at the periphery creating positive curvature, spanning a $z$-distance of $\sim 4 \mathrm{~nm}$ (Figure 4a). Positive curvature facilitating fusion is clearly evident at the periphery of the t-SNARE cluster (red areas in Figures $4 \mathrm{~b}$ and S10). Overall, the lipid tail order is much higher in the EC leaflet than in the IC leaflet, and lipids near the t-SNARE clusters are more disordered (Figure S11).
To validate the results obtained with the small t-SNARE fragment, we performed five additional clustering simulations (Sim4-8, each 5-10 $\mu$ s long) of $12 \mathrm{t}$-SNAREs that also included the entire SNARE domains as in the postfusion crystal structure. ${ }^{17}$ In these simulations, t-SNAREs also assembled into clusters (Figure 5a), but did so more slowly, and clusters

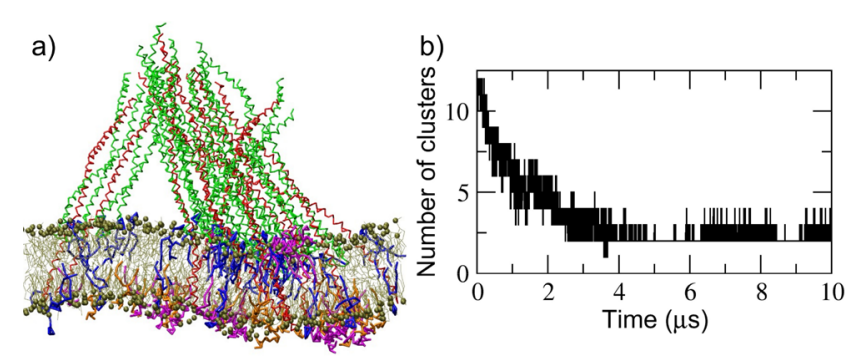

Figure 5. Clustering of t-SNARE with entire SNARE domain. (a) A snapshot at $10 \mu \mathrm{s}$, and (b) evolution of the number of t-SNARE clusters from one of the simulations (Sim6).

appeared more dynamic (Figure 5b), presumably due to the presence of the long and flexible SNARE domains hindering TM domain diffusion and interaction. Nevertheless, we observed a similar clustering of PIP2, GM lipids, and cholesterol colocalized with t-SNARE clusters. Curvature of the membrane associated with the clusters was also evident (Figure 5a).

This study provides molecular insights into the existence of liquid disordered nanodomains at the plasma membrane where the vesicles dock, waiting to fuse. The t-SNAREs form clusters of varying copy numbers. It has been shown that cholesterol induces clustering of stx and that PIP2 disperses these clusters. ${ }^{13,14}$ However, experimental and computational evidence also indicate that, in the absence of cholesterol, stx 1 clusters can be induced by PIP2. ${ }^{15}$ Here, we identify PIP2 in stx1/SNAP25 tSNARE clusters in the presence of cholesterol. Consistent with this result, a study in PC12 cells shows that PIP2 clusters apparently colocalize with t-SNARE clusters. ${ }^{24}$ In addition, we find that, in the EC leaflet, clusters of GM coexist with the stx1 clusters. The evidence from simulations on stx1-GM interactions has not been previously reported and needs to be assessed experimentally.

In summary, we find that t-SNARE clusters form lipid nanodomains forming an outward curvature around their center and an inward curvature near their edge. For membrane fusion, an initial contact between the two membranes must be established first. Intuitively, the contact site cannot be directly on top of the clusters, rather adjacent to them with fewer $t$ SNAREs present. Considering that only few SNAREs are required for fusion ${ }^{25}$ and that fusion occurs near the edge of $t$ SNARE clusters, ${ }^{6,11}$ we propose that t-SNARE clusters promote fusion by curving the membrane inward, toward the docked vesicle at the edge of $t$-SNARE clusters. The membrane bending, overcoming the separation between the membranes, will enable fusion induced by zippering of the SNARE domains. The local membrane curvature associated with the bending would also promote the formation of hydrophobic defects and lipid splay leading to the formation of a stalk. It is quite likely that the observed bending may be further advanced as the t-SNARE zippers with the vesicular synaptobrevin. In addition, the presence of other proteins such as synaptotagmins are thought to further increase the curvature and promote fusion. 


\section{ASSOCIATED CONTENT}

\section{S Supporting Information}

The Supporting Information is available free of charge on the ACS Publications website at DOI: 10.1021 /jacs.7b10677.

Details on computational methods, additional analysis, and lipid composition of membrane (PDF)

\section{AUTHOR INFORMATION}

\section{Corresponding Author}

*satyan.sharma@mpibpc.mpg.de

\section{ORCID}

Satyan Sharma: 0000-0001-5732-7002

Notes

The authors declare no competing financial interest.

\section{ACKNOWLEDGMENTS}

We are thankful for financial support from the European Research Council (ERC) (Grant ADG 322699) and from National Institutes of Health (NIH) grants R21NS088253 and R01GM121787. We thank the Max Planck Computing and Data Facility (MPCDF) for computational resources.

\section{REFERENCES}

(1) Weber, T.; Zemelman, B. V.; McNew, J. A.; Westermann, B.; Gmachl, M.; Parlati, F.; Söllner, T. H.; Rothman, J. E. Cell 1998, 92, 759-772.

(2) Hanson, P. I.; Heuser, J. E.; Jahn, R. Curr. Opin. Neurobiol. 1997, 7, $310-5$.

(3) Lang, T.; Halemani, N. D.; Rammner, B. Prog. Lipid Res. 2008, 47, 461-9.

(4) Ammar, M. R.; Kassas, N.; Chasserot-Golaz, S.; Bader, M. F.; Vitale, N. Front. Endocrinol. (Lausanne, Switz.) 2013, 4, 125.

(5) Fang, Q.; Lindau, M. Physiology 2014, 29, 278-85.

(6) Lang, T.; Bruns, D.; Wenzel, D.; Riedel, D.; Holroyd, P.; Thiele, C.; Jahn, R. EMBO J. 2001, 20, 2202-13.

(7) Knowles, M. K.; Barg, S.; Wan, L.; Midorikawa, M.; Chen, X.; Almers, W. Proc. Natl. Acad. Sci. U. S. A. 2010, 107, 20810-5.

(8) Lu, X.; Zhang, Y.; Shin, Y. K. Nat. Struct. Mol. Biol. 2008, 15, 700-6.

(9) Barg, S.; Knowles, M. K.; Chen, X.; Midorikawa, M.; Almers, W. Proc. Natl. Acad. Sci. U. S. A. 2010, 107, 20804-9.

(10) Gandasi, N. R.; Barg, S. Nat. Commun. 2014, 5, 3914.

(11) Ullrich, A.; Bohme, M. A.; Schoneberg, J.; Depner, H.; Sigrist, S. J.; Noe, F. PLoS Comput. Biol. 2015, 11, e1004407.

(12) Sieber, J.J.; Willig, K. I.; Kutzner, C.; Gerding-Reimers, C.; Harke, B.; Donnert, G.; Rammner, B.; Eggeling, C.; Hell, S. W.; Grubmuller, H.; Lang, T. Science 2007, 317, 1072-6.

(13) Murray, D. H.; Tamm, L. K. Biochemistry 2009, 48, 4617-25.

(14) Murray, D. H.; Tamm, L. K. Biochemistry 2011, 50, 9014-22.

(15) van den Bogaart, G.; Meyenberg, K.; Risselada, H. J.; Amin, H.; Willig, K. I.; Hubrich, B. E.; Dier, M.; Hell, S. W.; Grubmüller, H.; Diederichsen, U.; Jahn, R. Nature 2011, 479, 552-5.

(16) Monticelli, L.; Kandasamy, S. K.; Periole, X.; Larson, R. G.; Tieleman, D. P.; Marrink, S.-J. J. Chem. Theory Comput. 2008, 4, 819834.

(17) Stein, A.; Weber, G.; Wahl, M. C.; Jahn, R. Nature 2009, 460, 525-528.

(18) Breckenridge, W. C.; Gombos, G.; Morgan, I. G. Biochim. Biophys. Acta, Biomembr. 1972, 266, 695-707.

(19) Sharma, S.; Kim, B. N.; Stansfeld, P. J.; Sansom, M. S.; Lindau, M. PLoS One 2015, 10, e0144814.

(20) Fujimoto, T.; Parmryd, I. Front Cell Dev Biol. 2016, 4, 155.

(21) Weber, P.; Batoulis, H.; Rink, K.; Dahlhoff, S.; Pinkwart, K.; Sollner, T. H.; Lang, T. eLife 2017, 6, e19394.
(22) Ingolfsson, H. I.; Melo, M. N.; van Eerden, F. J.; Arnarez, C.; Lopez, C. A.; Wassenaar, T. A.; Periole, X.; de Vries, A. H.; Tieleman, D. P.; Marrink, S. J. J. Am. Chem. Soc. 2014, 136, 14554-9.

(23) Sonnino, S.; Mauri, L.; Chigorno, V.; Prinetti, A. Glycobiology 2007, 17, 1R-13R

(24) Aoyagi, K.; Sugaya, T.; Umeda, M.; Yamamoto, S.; Terakawa, S.; Takahashi, M. J. Biol. Chem. 2005, 280, 17346-52.

(25) Mohrmann, R.; Sorensen, J. B. J. Mol. Neurosci. 2012, 48, 387-94. 